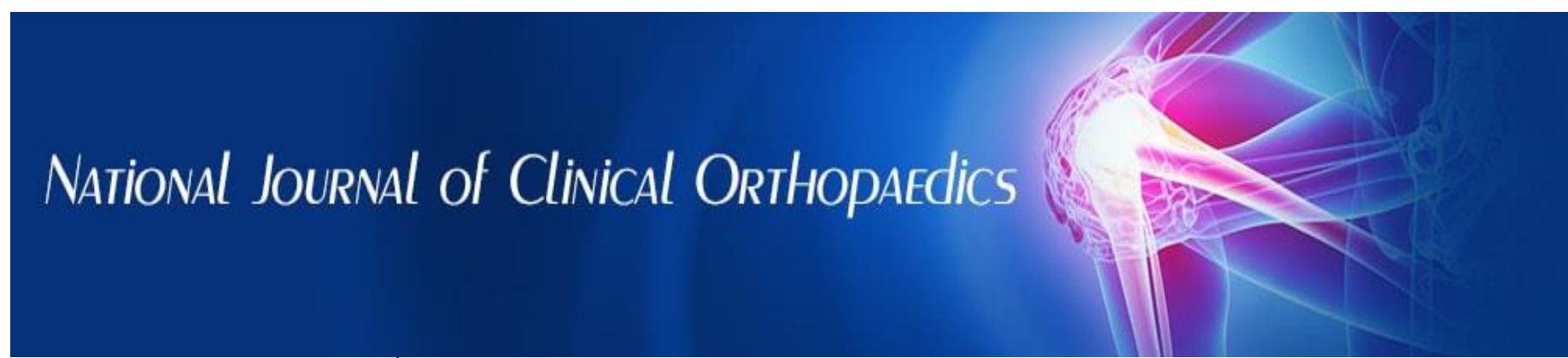

ISSN (P): 2521-3466

ISSN (E): 2521-3474

(C) Clinical Orthopaedics

www.orthoresearchjournal.com

2020; 4(1): 85-89

Received: 06-11-2019

Accepted: 10-12-2019

Dr. Rajesh Kumar Sharma Senior Resident Doctor,

Department of Orthopaedics, SMS Medical College, Jaipur

(Rajasthan) \& Former

Orthopaedic Resident, Govt.

Medical College, Kota

Rajasthan, India

Dr. Rajesh Goel

Sr. Professor \& Head of

Department, Department of Orthopaedics, Govt. Medical

College, Kota Rajasthan and President of Rajasthan Orthopaedic Surgeons Association, Kota Rajasthan, India

Corresponding Author: Dr. Rajesh Kumar Sharma Senior Resident Doctor, Department of Orthopaedics, SMS Medical College, Jaipur (Rajasthan) \& Former Orthopaedic Resident, Govt. Medical College, Kota

Rajasthan, India

\section{Early functional results of minimally invasive subvastus approach in primary total knee arthroplasty: A randomized controlled study}

\author{
Dr. Rajesh Kumar Sharma and Dr. Rajesh Goel
}

DOI: https://doi.org/10.33545/orthor.2020.v4.i1b.203

\section{Abstract}

Background: Minimally invasive subvastus approach preserves the quadriceps extenser mechanism, reduces postoperative pain, provides early mobilization and helps in faster recovery. Our randomized controlled study presents the early functional results of minimally invasive subvastus approach in primary total knee arthroplasty (TKA).

Materials and Methods: Primary TKA procedures were done in 50 patients via minimally invasive subvastus approach and in another 50 patients via medial parapatellar approach, randomly. The postoperative clinical evaluation was done at day $0,1,3$ and at discharge. All categorical variables were analysed by the null hypotheses of no differences in patients treated with either group at $95 \%$ significance level $(P$-value $<0.05)$.

Results: In subvastus group, the average length of cutaneous incision was shorter and less perioperative blood loss as compared to the medial parapatellar group. The average surgical time was significantly more in subvastus group. The VAS scores were significantly lower in subvastus group at day 1 and day 3. Time to straight leg raise, ability to use commode chair, and stairs climbing were significantly earlier (P-value $<0.05$ ) in subvastus group. The average hospital stay was 2.24 days shorter in subvastus group. Conclusion: With a learning curve, minimally invasive subvastus approach produces significantly less pain, early rehabilitation, and shorter hospital stay, without increase in the overall complication rate.

Keywords: Minimally invasive subvastus approach, medial parapatellar approach, Total knee

\section{Introduction}

Minimally invasive and muscle sparing techniques for primary total knee arthroplasty (TKA) are gaining popularity due to less postoperative pain, less bleeding and more rapid rehabilitation. Here we are discussing about the early fuctional results of minimally invasive subvastus approach. According to literature ${ }^{[1,2]}$ the ultimate functional results of subvastus approach are comparable with medial parapatellar approach, but length of recovery period is shorter in subvastus approach ${ }^{[3,4]}$ due to preservation of extensor mechanism of knee. However, it has some limitations with obese patients and stiff knees, and has a learning curve for surgeons ${ }^{5}$.

\section{Materials and Methods}

This study was conducted in the department of Orthopaedics, Govt. Medical College and attached group of hospitals, Kota (Rajasthan) during the years 2015-2018. After approval of local Medical Ethics Committee, a prospective, randomized trial was designed with sample size of 100 patients treated with primary TKA. Written informed consent was taken from all patients involved in our study. These patients were randomized into two groups of 50 patients each, group A (subvastus group) and group B (medial parapatellar group) with inclusion criteria: primary severe osteoarthritis of knee and willingness to participate in the trial and exclusion criteria: any previous knee surgery or osteotomy around knee, ongoing knee sepsis, rheumatoid arthritis, flexion deformity of knee $>15$ degree, varus-valgus deformity $>15$ degree, knee flexion $<90$ degree, BMI $>30 \mathrm{~kg} / \mathrm{m}^{2}$, and muscular weakness. Same type of implant (PCL sacrificing LCS design of DePuy, Zimmer, and Stryker) 
was used in both groups. All procedures were performed by the same experienced Arthroplasty surgeon under combined spinal epidural Anesthesia. All demographic data (age, gender, BMI and comorbidities) were comparable in both groups. Similar pain management, and same physiotherapy protocol, (Postop day 0 - static quadriceps exercises, flexion and extension exercises of knee, straight leg raising exercises; Postop day 1bed side mobilisation and waking with walker; Postop day 2commode chair training; Postop day 3- stairs climbing exercises) received by each group. Although, in case of increased pain and intolerance, the physiotherapy protocols were somewhat delayed. In each group the VAS scores (scale of 0 to 10) were recorded both at rest and at movement on day $0,1,3$ and at discharge. Time of SLR, ability to stand with walker, ability to use a commode chair, ability to climb stairs, hospital stay and knee flexion at discharge were also compared in both the groups. Each patient was discharged when VAS below two and was comfortable to use commode chair. Length of cutaneous incision, surgical time and perioperative blood loss were also compared in both groups. Removal of drain was done after 24 hours of surgery and postoperative blood loss was estimated.

\subsection{Statistical Analysis}

The statistical analysis was carried out using SPSS 16.0 and MS Excel 2013. All categorical variables were analysed by the null hypotheses of no differences in patients treated with either group. The difference was considered statistically significant at 95\% significance level $(P$-value $<0.05)$. The unpaired $t$-test was used to evaluate possible statistical differences of values within and between the both groups as well.

\subsection{Minimally invasive subvastus approach}

The patient was positioned supine in the standard fashion. The leg was exsanguinated with the use of an Esmarche, and the tourniquet was inflated with the knee flexed to at least 90 degrees to prevent tenodesis of the extensor mechanism. The knee arthrotomy started with a straight anterior skin incision, beginning $8 \mathrm{~cm}$ above the patella, extended it distally just medial and $2 \mathrm{~cm}$ distal to the tibial tubercle. Then superficial fascia was incised slightly medial to the patella and bluntly dissected it off the vastus medialis muscle fascia down to the muscle insertion. Inferior edge of the vastus medialis was identified and bluntly dissected it off the periosteum and intermuscular septum for a distance of $10 \mathrm{~cm}$ proximal to the adductor tubercle. The tendinous insertion of the muscle on the medial patellar retinaculum was identified and the vastus medialis muscle was lifted anteriorly and L-shaped arthrotomy was done via vastus insertion on the medial patellar retinaculum and upto medial edge of the patella. The medial edge of the patellar tendon was partially released and the patella was everted laterally with the knee extended (Campbell). Further, the distal femur and proximal tibia were prepared, and the prosthesis was inserted. A standard closure was done at the end of the surgery.

\section{Results}

In our study, the mean length of cutaneous incision in Group A (subvastus group) and Group B (medial parapatellar group) were 12.15 and $13.96 \mathrm{~cm}$, respectively [Table 1 , and Figure 1] and the difference was not statistically significant ( $P$-value 0.7385$)$. The mean estimated blood loss in Group A (subvastus group) and Group B (medial parapatellar group) were 416.75 and 453.86 $\mathrm{mL}$, respectively [Table 1 , and Figure 1] and the difference was not statistically significant ( $P$-value 0.4064$)$. The mean surgical time required for subvastus approach and medial parapatellar approach [Table 1, and Figure 1] were 105.9 and $88.3 \mathrm{~min}$, respectively and the difference was statistically significant $(P$ value $<0.01$ ).

In this study, the mean VAS scores at rest in Group A (subvastus group) on day 0 , day 1 , day 3 , and discharge were $4.35,3.11$, 2.16, and 1.57, respectively; while at movement were $6.12,4.1$, 3.12, and 2.1, respectively [Table 2 and Figure 2]. Similarly, the mean VAS scores at rest in Group B (medial parapatellar group) on day 0 , day 1 , day 3 , and discharge were $4.4,3.97,2.99$, and 1.63, respectively; while at movement were $6.23,5.44,4.28$, and 2.23, respectively [Table 2 and Figure 2]. The differences in mean VAS scores on day 0 between both the groups were not statistically significant ( $P$-value 0.1633 at rest \& 0.1834 at movement). The differences in mean VAS scores on day 1 and day 3 were statistically significant $(P$-value $<0.05)$ both at rest and at movement. At discharge the differences in mean VAS scores were not statistically significant in both conditions in both groups but more importantly, the discharge in subvastus group was 2.24 mean days earlier than the medial parapatellar group [Table 3 and Figure 3].

The ability to perform straight leg raise (SLR) postoperatively was earlier [Table 3 and Figure 3] in the subvastus group (mean days 1.77 as compared to 2.19 in medial parapatellar group) which was statistically significant $(P$-value $<0.01)$. Although, time to stand with walker in subvastus group was statistically not significant $(P$-value $=1.095)$ as compared to medial parapatellar group. The ability to use commode chair and ability to climb stairs, both were early [Table 3 and Figure 3] in the subvastus group and differences of both outcomes were statistically significant $(P$-value $<0.05)$. The mean knee flexion at the time of discharge in subvastus and medial parapatellar group were $98.2^{\circ}$ and $94.7^{\circ}$, respectively [Table 3] and the difference was statistically not significant ( $P$-value 0.7628$)$.

Table 1: Mean length of cutaneous incision, and mean surgical time, and mean estimated blood loss.

\begin{tabular}{|c|c|c|c|}
\hline & Mean length of Cutaneous Incision (cm) & Mean Surgical time (minutes) & Mean Estimated Blood loss (ml) \\
\hline Subvastus group (A) & 12.15 & 105.9 & 416.75 \\
\hline Medial parapatellar group (B) & 13.96 & 88.3 & 453.86 \\
\hline
\end{tabular}




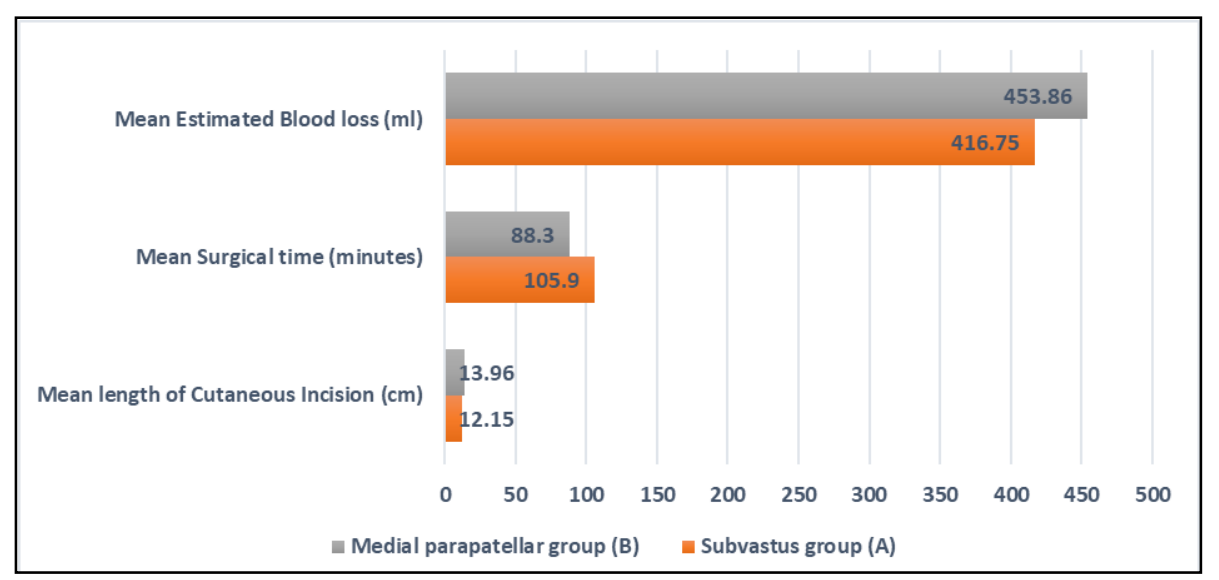

Fig 1: Graph showing mean length of cutaneous incision, and mean surgical time, and mean estimated blood loss between subvastus and medial parapatellar group.

Table 2: Mean VAS scores (scale 0-10).

\begin{tabular}{|c|c|c|c|c|c|}
\hline \multicolumn{2}{|c|}{} & Day 0 & Day 1 & Day 3 & On Discharge \\
\hline \multirow{2}{*}{ Subvastus Group (A) } & At Rest & 4.35 & 3.11 & 2.16 & 1.57 \\
\cline { 2 - 6 } & With Movement & 6.12 & 4.1 & 3.12 & 2.1 \\
\hline \multirow{2}{*}{ Medial parapatellar group (B) } & At Rest & 4.4 & 3.97 & 2.99 & 1.63 \\
\cline { 2 - 6 } & With Movement & 6.23 & 5.44 & 4.28 & 2.23 \\
\hline
\end{tabular}

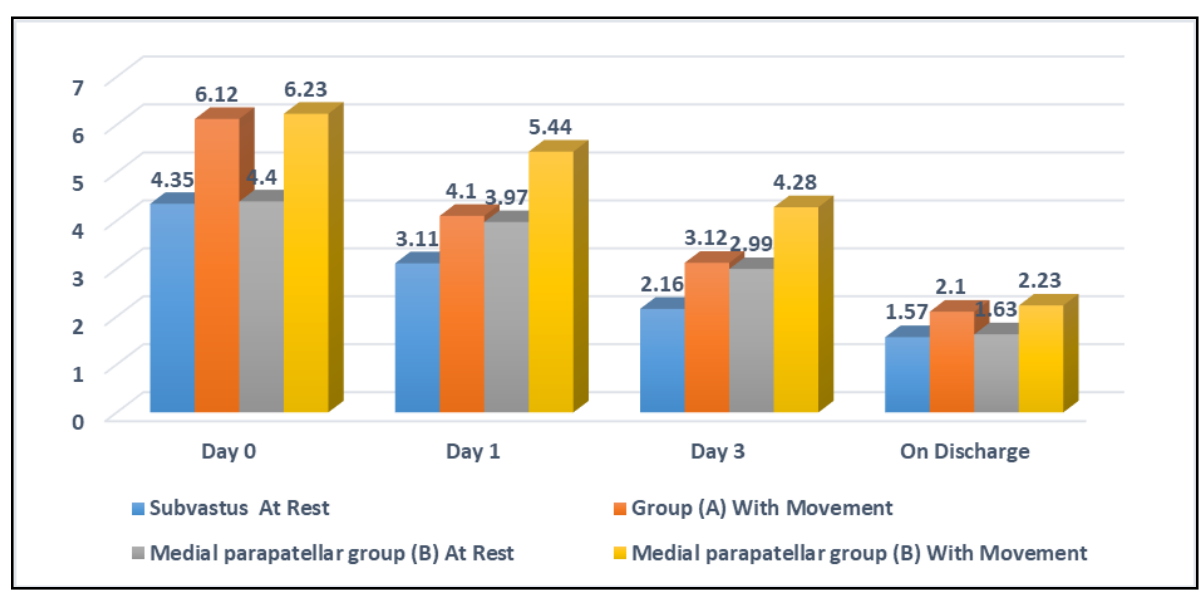

Fig 2: Graph showing mean VAS scores (scale 0-10) between subvastus and medial parapatellar group.

Table 3: Early fuctional outcomes (Mean days)

\begin{tabular}{|c|c|c|c|c|c|c|}
\hline & Time to SLR* & $\begin{array}{c}\text { Ability to stand } \\
\text { with walker }\end{array}$ & $\begin{array}{c}\text { Ability to use a } \\
\text { commode chair }\end{array}$ & $\begin{array}{c}\text { Day of } \\
\text { Discharge }\end{array}$ & $\begin{array}{c}\text { Knee flexion at } \\
\text { Discharge (degree) }\end{array}$ & $\begin{array}{c}\text { Ability to } \\
\text { climb stairs }\end{array}$ \\
\hline Subvastus group (A) & 1.77 & 1.83 & 3.52 & 3.68 & 98.2 & 4.18 \\
\hline Medial parapatellar group (B) & 2.19 & 1.91 & 5.71 & 5.92 & 94.7 & 6.42 \\
\hline
\end{tabular}

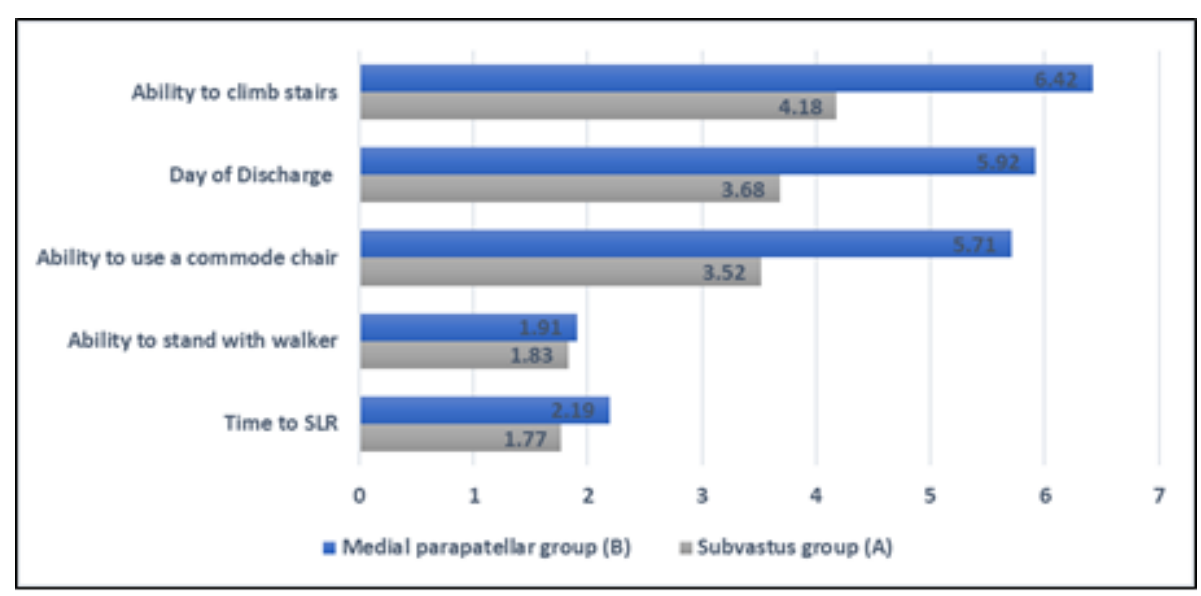

Fig 3: Graph showing various early functional outcomes between and subvastus and medial parapatellar group. 


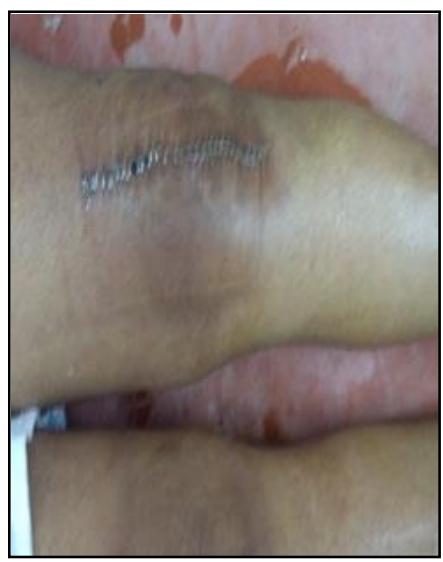

Cutaneous Incision

*(SLR- Straight Leg Raising)

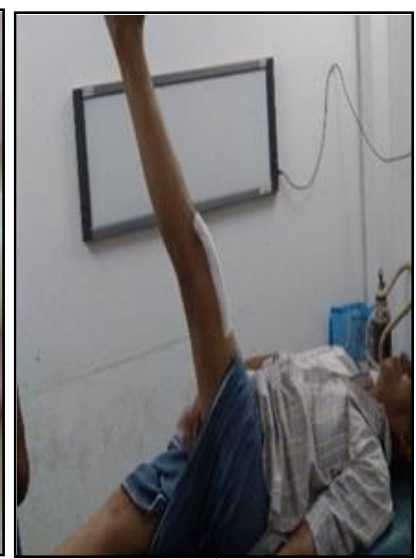

SLR ${ }^{*}$

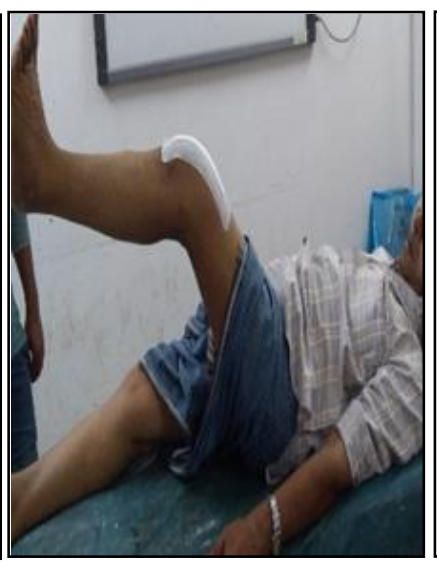

Knee flexion

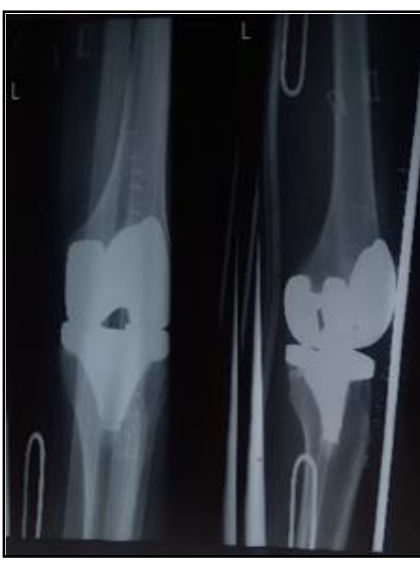

AP \& Lateral views

Fig 4: Clinical \& radiological evaluation of knee in subvastus group at the time of discharge.

Our study doesn't show any major perioperative complication in either group. During surgery, being more careful with the soft tissues may have avoided the high number of cutaneous complications.

\section{Discussion}

Surgical techniques that reduce injury to the knee extensor mechanism may cause less pain and faster rehabilitation ${ }^{[6]}$ after primary total knee arthroplasty (TKA). The most common TKA technique, as reported in national joint registries, is medial parapatellar approach ${ }^{[7]}$ as it provides extensile exposure. But it causes impairment of blood supply to the patella that may leads to osteonecrosis of patella, fracture of patella or anterior knee pain ${ }^{[8,9]}$.

Minimally invasive subvastus (SV) approach was introduced by Hoffman et al ${ }^{[10]}$. This approach can conserve the blood supply to the patella, even if lateral retinacular release is required, and in addition, it provides an adequate surgical exposure for TKA. Furthermore, it reduces the problem of patellar tracking and early postoperative rehabilitation by preserving the quadriceps muscle function. The early quadriceps recovery, and short hospital stay make it a good alternative to the medial parapatellar approach for primary TKA. Although, sometimes in subvastus approach patellar eversion may be difficult and knee exposure may be inadequate specially in obese patients with excessive thigh girth and stiff knees ${ }^{[10]}$.

In our study, the average length of cutaneous incision was shorter and perioperative blood loss was less in subvastus group as compared to the medial parapatellar group but this difference was statistically insignificant. The average surgical time in subvastus approach was significantly more than medial parapatellar approach because this technique is demanding and has along learning curve. All these findings correlate with the study of Hongsen Chiang et al. ${ }^{[11]}$.

The VAS scores were significantly lower in subvastus group at day 1 and day 3 due to less injury to the knee extensor mechanism. The average flexion at the time of discharge in subvastus and parapatellar group were 98.2 and $94.7^{\circ}$, respectively. In the literature, these findings are comparable with the findings of Ahmed M. Kholeif et al. ${ }^{[12]}$.

Though time to stand with walker was same for both groups, but other functional outcomes such as SLR, ability to use commode chair, and stairs climbing were also significantly earlier in subvastus group. It shows faster rehabilitation due to better quadriceps strength ${ }^{[4,13]}$. The discharge of the patient was 2.24 mean days earlier in subvastus group. This shorter hospital stay leads to somewhat less expenditure of the surgery and improves patient satisfaction. Our study findings correlate with study of Munenori et al ${ }^{[14]}$.

\section{Conclusion}

Postoperative pain and delayed mobilization limit the early rehabilitation after medial parapatellar approach in primary TKA. Our study shows that subvastus approach produce significantly less pain, faster rehabilitation, shorter hospital stay, less expenditure, and more patient satisfaction without increase in the overall complication rate as compared to medial parapatellar approach. Although, it is technically demanding and has a learning curve.

\section{References}

1. Cila E, Güzel V, Ozalay M, Tan J, Sims ek SA, Kanatli U et al. Subvastus versus medial parapatellar approach in total knee arthroplasty. Arch Orthop Trauma Surg. 2002; 122:65-8.

2. Fauré BT, Benjamin JB, Lindsey B, Volz RG, Schutte D. Comparison of the subvastus and paramedian surgical approaches in bilateral knee arthroplasty. J Arthroplasty. 1993; 8:511-6.

3. Dutka J, Skowronek M, Sosin P, Skowronek P. Subvastus and medial parapatellar approaches in TKA: Comparison of functional results. Orthopedics. 2011; 34:148.

4. Roysam GS, Oakley MJ. Subvastus approach for total knee arthroplasty: A prospective, randomized, and observer-blinded trial. J Arthroplasty. 2001; 16:454-7.

5. King J, Stamper DL, Schaad DC, Leopold SS. Minimally invasive total knee arthroplasty compared with traditional total knee arthroplasty. Assessment of the learning curve and the postoperative recuperative period. J Bone Joint Surg Am. 2007; 89(7):1497-503.

6. Bridgman SA, Walley G, Clement D, Griffiths D, Maffulli N. Subvastus approach is more effective than a medial parapatellar approach in primary total knee arthroplasty: a randamized controlled trial. Knee. 2009; 16(3);216-22. Epub 2009 Jan 09.

7. Bourke MG, Buttrum PJ, Fitzpatrick PL, Dalton PA, Jull GA, Russell TG et al. Systematic review of medial parapatellar and subvastus approaches in total knee arthroplasty. J Arthroplasty. 2010; 25(5):728-34. Epub 2009 Aug 18.

8. Holtby RM, Grosso P. Osteonecrosis and resorption of the patella after total knee replacement: a case report. Clin 
Orthop Relat Res. 1996; 328:155-158

9. Scapinelli R. Blood supply of the human patella. Its relation to ischaemic necrosis after fracture. J Bone Joint Surg Br. 1967; 49:563-570

10. Hofmann AA, Plaster RL, Murdock LE. Subvastus (southern) approach for primary total knee arthroplasty. Clin Orthop Relat Res. 1991; 269:70-77

11. Hongsen Chiang, Chung-Chien Lee, Wei-Peng Lin, ChingChuan Jiang. Comparison of quadriceps-sparing minimally invasive and medial parapatellar total knee arthroplasty: A 2-year follow-up study. Journal of Formosan Medical Asso. 2012; 111:698-704.

12. Ahmed M Kholeif M.D, Yaser A Radhan, M.D, Ali M Mansour M.D, Hesham Ali H Al-Moalem M.D. Subvastus approach is a good alternative for primary TKA without increase in complication rate. Med. J Cairo Univ. 2017; 85(2):575-582.

13. Matsueda M, Gustilo RB. Subvastus and medial parapatellar approaches in total knee arthroplasty. Clin Orthop Relat Res. 2000; 371:161-8.

14. Munenori Mutsuedu, Ramon B. Gustilo: Subvastus and Medial Parapatellar Approaches in Total Knee Arthroplasty, Clinical Orthopeadics and releated research, Number. 2000; 371:161-8. 